\title{
Interior structura variations in the secondary components of two Algol-type eclipsing binary systems: SW Cygni and RR Draconis
}

\author{
S. B. Qian ${ }^{1,2}$, L. Y. Zhu ${ }^{1,2}$, and S. Boonrucksar ${ }^{3}$ \\ 1 Yunnan Observatory, National Astronomical Observatories, Chinese Academy of Sciences, PO Box 110, 650011 Kunming, \\ PR China \\ 2 United Laboratory of Optical Astronomy, Chinese Academy of Science (ULOAC), PR China \\ 3 Astronomy Research unit, Department of Physics, Faculty of Science, Chiang Mai University, Chiang Mai 50200, Thailand
}

Received 17 July 2002 / Accepted 23 September 2002

\begin{abstract}
SW Cyg and DD Dra are two Algol-type eclipsing binary systems. Both contain an A2-type main-sequence primary and a K0-type secondary component with a rather deep primary light minimum in their light curves ( 2.6 and 3.8 , respectively). In the present paper, orbital period changes of these stars are studied based on the analyses of their century-long times of light minimum. Several period jumps are found to superimpose on a rapid secular increase $\left(\mathrm{d} P / \mathrm{d} t=+4.71 \times 10^{-6}\right.$ and $+4.24 \times$ $10^{-6}$ days/year, respectively). Since the period increases are rather rapid, the mass exchange between the components would be dynamical. SW Cyg is a low-mass ratio system $(q=0.20)$ and it is expected that the mass ratio of RR Dra may also be very low because of the rather deep primary eclipse minimum. The rapid period increases suggest that a rapid mass transfer also occurs in a low-mass ratio Alogl-type close binary which is opposite to the theoretically evolutionary scheme of the binary star. The period jumps superimposed on the long-term increases are caused by the structura variation of the cool mass-loser via instabilities in the convective outer layer (COL) or via cyclic magnetic activity of the K0-type components. As the period jumps do not occur in a short-term alternating way, the structura change caused by instabilities in the COL via a dynamical mass loss from the cool subgiant may be more plausible.
\end{abstract}

Key words. stars: binaries: close - stars: individual: SW Cyg - stars: individual: RR Dra - stars: evolution

\section{Introduction}

SW Cyg is an Algol-type eclipsing binary that contains an A2-type main-sequence primary star and a K0-type subgaint that fills its Roche lobe and transfers mass to its companion. The principal eclipse is very deep and total. The light is constant at the bottom of primary minimum for nearly three hours. Spectroscopic elements of the binary star were published by Struve (1946) who found a rapidly rotating gaseous ring and disk around the primary component. Photometric light curves and solutions of the system were published by Walter (1971), Hall \& Garrison (1972), Hall et al. (1979), Olson (1982), and by Wilson \& Garrison (1988).

The variability of the period of SW Cyg has been studied by various authors. Dugan \& Wright (1939) found the period to be variable and attribute the change to the presence of a third body. Subsequently, by considering that the observed times of light minimum deviated remarkably from the predictions based on this hypothesis and that the solutions of the light-time effect suggest a very large mass of the third body $\left(m_{3}=8 M_{\odot}\right)$, Frieboes-Conde \& Herczeg (1973) pointed out that the period variations were formed by a set of sudden, discontinuous

Send offprint requests to: $\mathrm{S}$. Qian, e-mail: qsb@netease.com changes. The period changes of the system were later explained in terms of the model of Biermann \& Hall (1973) by Chaubey (1980) who showed data after 1910 indicating a continuous period increase. In their period study of SW Cyg, a cyclic variation with a period of 96 years was proposed by Berrington \& Hall (1994), which was interpreted as magnetic cycle in the K0type subgiant. However, in a recent study by Šimon (1997a), it was discovered that the episodes of period decrease are accompanied by minimum of brightness of the mass-loser, which indicates that the period change may be due to instabilities in the outer convective layer of the mass-loser.

The Algol-type eclipsing binary, RR Dra, resembles some observed properties of SW Cyg. It also contains an A2-type main-sequence primary and a K0-type secondary (Yoon et al. 1994). The primary light minimum is so deep as to disappear from view in the seven-inch equatorial during the eclipses (Seares 1912). The light is constant at the bottom of the primary light minimum for a duration of $d=0.06$ (Shapley 1913). The orbital period change of RR Dra was first announced Nijland (1917) who proposed a parabolic change of the $\mathrm{O}-\mathrm{C}$ curve. The variation was subsequently confirmed by Dugan \& Wright (1939) but showed that the parabolic term introduced by Nijland must be abandoned. Their $\mathrm{O}-\mathrm{C}$ curve 
indicated a periodic change. Since the period study of Dugan \& Wright (1939), 63 years have elapsed. Many times of light minimum have been published, but a period study is absolutely required. In the present paper, the times of minimum light of the two binaries, SW Cyg and RR Dra, are compiled and the variations in the orbital periods are studied in more detail. Then, the structure and evolutionary states of both binary stars are discussed.

\section{Orbital period variation of SW Cyg}

In order to form the $\mathrm{O}-\mathrm{C}$ curve and to study the period change of SW Cyg, a careful search of all available times of light minimum is made. Many eclipse times were compiled by Dugan \& Wright (1939), Frieboes-Conde \& Herczeg (1973) and by Berrington \& Hall (1994). 101 times of light minimum were compiled at the Eclipsing Binary Minima Database (available at http://www.oa.uj.edu.pl/ktt/krttk_dn.html). Two photoelectric timings, HJD 2444428.7635 and 2444460.7750, by Olson (1981) were not included in those compilations. The $\mathrm{O}-\mathrm{C}$ values are computed with the following linear light elements given by Dugan \& Wright (1939):

$\operatorname{MinI}=2418440.758+4.5727923 \times E$

and are displayed graphically against Julian date in Fig. 1. During the calculation of the $\mathrm{O}-\mathrm{C}$ values, several times of light minimum, obtained from the EBMD, with the same epoch are averaged and only the mean $\mathrm{O}-\mathrm{C}$ values are plotted in Fig. 1. Two EBMD data points, HJD 2449175.000 and HJD 2449198.000, were discarded since their O-C values show large deviations when compared to the general trend formed by the other eclipse times.

The $\mathrm{O}-\mathrm{C}$ plot in Fig. 1 shows that the variation of the $\mathrm{O}-\mathrm{C}$ values is rather complex. By considering a secular period increase and using weights 1 to the visual and photographic data and 8 to the photoelectric observations, a weighted leastsquares solution leads to the following quadratic ephemeris with the mean error for each term:

$$
\begin{aligned}
\text { MinI }= & 2418440.669(27)+4.57272811(16) \\
& \times E+2.95(2) \times 10^{-8} \times E^{2} .
\end{aligned}
$$

With the quadratic term of the ephemeris, a continuous period increase with a rate of $\mathrm{d} P / \mathrm{d} t=+4.71 \times 10^{-6}$ days/year is determined. As displayed in Fig. 1, the quadratic ephemeris can give a good fit to the mean trend of the $\mathrm{O}-\mathrm{C}$ curve but without describing any particular characteristics.

The residuals $\left((\mathrm{O}-\mathrm{C})^{\prime}\right.$ values $)$ from the quadratic ephemeris (2) are plotted in Fig. 2. As shown in this figure, the $(\mathrm{O}-\mathrm{C})^{\prime}$ curve can be divided into several portions indicating that, apart from the secular long-term increase, several sudden jumps have occurred in the orbital period of SW Cyg in a time interval of 102 years. Between those jumps the orbital period is assumed to have undergone a steady increase. With the least-squares method, the values of $\Delta T$ and $\Delta P$ in the following function:

$(\mathrm{O}-\mathrm{C})^{\prime}=\Delta T+\Delta P \times E$

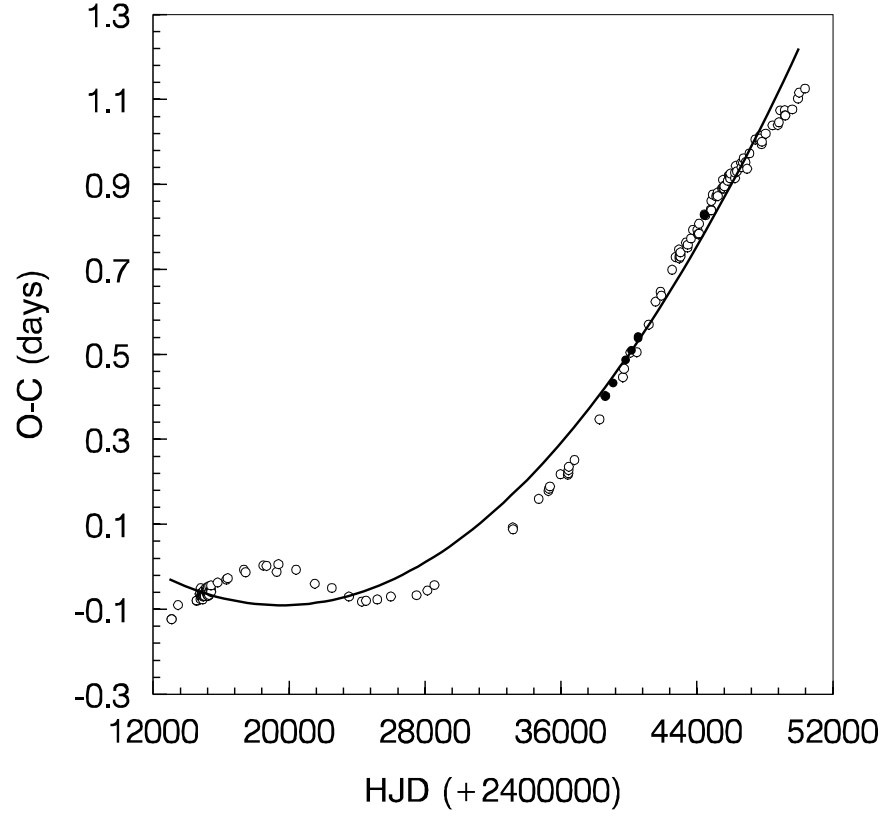

Fig. 1. The century-long O-C curve of SW Cyg with respect to the ephemeris of Dugan \& Wright. The solid line refers to the best fit parabola to the data (Eq. (2)). Open circles represent to visual or photographic data and solid refer to photoelectric observations.

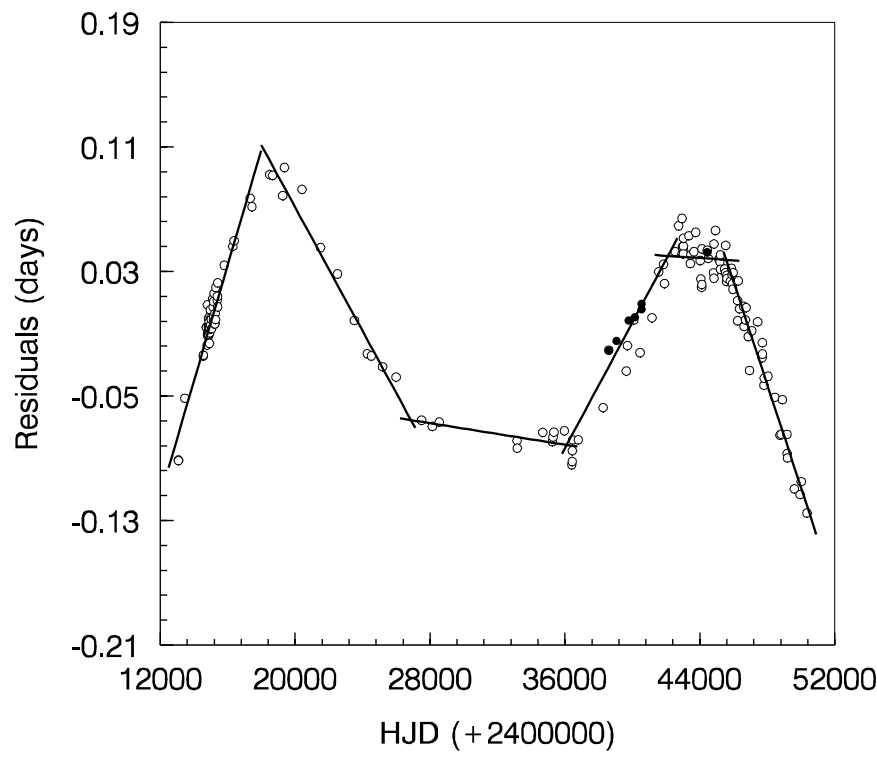

Fig. 2. Residuals in days of SW Cyg after subtracting the best-fit parabola given in Eq. (2). Solid lines represent the best description with several linear ephemerides.

are calculated for each portion and are listed in Table 1. Then, the orbital period of SW Cyg, at any cycle $E$, can be computed with the equation:

$P(E)=P_{\mathrm{e}}+\Delta P+\frac{\mathrm{d} P}{\mathrm{~d} t} \times \frac{E P}{365.24}$

where $P_{\mathrm{e}}=4.5727923$ is the ephemeris period. The results are shown in Fig. 3, where the difference between the real period $P(E)$ and the ephemeris period $P_{\mathrm{e}}$, in units of $10^{-5}$ days, are plotted as a function of time. Five irregular period jumps 


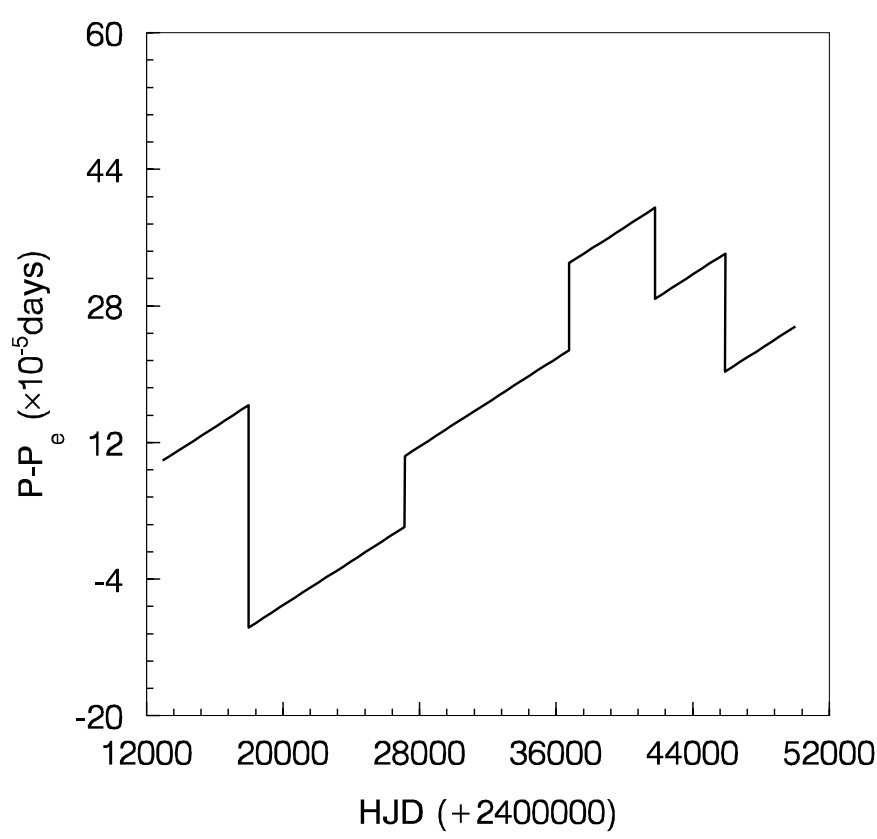

Fig. 3. Orbital period change of SW Cyg in $10^{-5}$ days as a function of time.

Table 1. Several irregular period jumps for SW Cyg.

\begin{tabular}{lll}
\hline \hline Interval of cycles & $\Delta T$ (days) & $\Delta P\left(10^{-5}\right.$ days $)$ \\
\hline-1200 to -100 & $+0.1245( \pm 0.0050)$ & $+17.0( \pm 0.67)$ \\
-100 to +1900 & $+0.1022( \pm 0.0049)$ & $-9.09( \pm 0.51)$ \\
+1900 to +4000 & $-0.0510( \pm 0.0083)$ & $-0.79( \pm 0.24)$ \\
+4000 to +5100 & $-0.4383( \pm 0.0348)$ & $+9.24( \pm 0.76)$ \\
+5100 to +6000 & $+0.1100( \pm 0.0348)$ & $-1.26( \pm 0.62)$ \\
+6000 to +6900 & $+0.9341( \pm 0.0381)$ & $-15.1( \pm 0.60)$ \\
\hline
\end{tabular}

occurred around HJD 2418000, HJD 2427100, HJD 2436700 , HJD 2441800, and HJD 2445900.

\section{Orbital period variation of RR Dra}

Many times of light minimum of RR Dra were collected by Dugan \& Wright (1939). After their collection, some eclipse times were published by Szczepanowska $(1955,1956,1959)$ and by Whitney (1957). 115 timings published in several journals (BBASG Bull., AN, MVS, and BRNO) were compiled at the EBMD and two photoelectric minima, HJD 2451315.4247 and 2451414.5215, were published by Agerer et al. (2001). The O-C curve calculated with the following linear ephemeris given by Dugan \& Wright (1939):

MinI $=2417026.680+2$ d. $83110 \times E$

are presented graphically against Julian date in Fig. 4. During the calculation of the $\mathrm{O}-\mathrm{C}$ residuals, 4 EBMD data points, HJD 2441555.392, 2441555.398, 2447456.360, and 2449568.495, were discarded, and some EBMD timings with the same epoch were averaged.

The general O-C trend displayed in Fig. 4 shows an upward parabolic variation suggesting a secular period increase for the system. By using the same weights as those used for SW Cyg, a

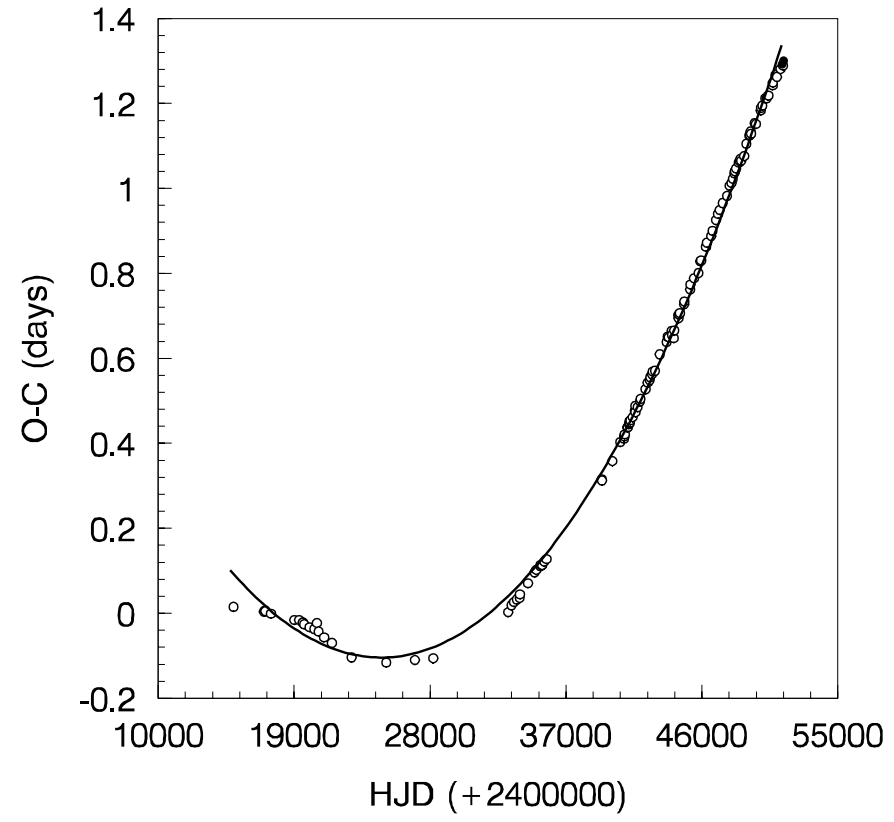

Fig. 4. The same as Fig. 1, but for RR Dra.

weighted least-squares solution yields the following quadratic ephemeris:

$$
\begin{gathered}
\text { Min.I }=\text { HJD 2417026.6990(43)+2.83100995(7) } \\
\quad \times E+1.64(1) \times 10^{-8} \times E^{2}
\end{gathered}
$$

and a rapid period increase rate: $\mathrm{d} P / \mathrm{d} t=+4.24 \times$ $10^{-6}$ days/year. As displayed in Fig. 4, this quadratic ephemeris can fit the general trend of the $\mathrm{O}-\mathrm{C}$ curve very well.

The residuals from the quadratic ephemeris (6) are displayed in Fig. 5, which show that the variation of these residuals is not continuous. This indicates that, apart from the secular rapid increase, several irregular jumps have taken place in the orbital period of RR Dra within a time interval of 100 years between late 1899 and 1999. By using the same method as that used for SW Cyg, the values of $\Delta T$ and $\Delta P$ for each segment are computed and are listed in Table 2. The orbital period at any cycle $E$ is calculated with Eq. (4). Finally, the period changes are displayed against Julian date in Fig. 6, where the difference between the real period and the ephemeris period Pe (2.8311 days) is plotted.

\section{Discussions and conclusions}

The observed properties of the two Algol-type binary stars, SW Cyg and RR Dra, are nearly the same. Both contain an A2-type main-sequence primary and a K0-type secondary component, and undergo well-defined deep complete eclipses (see Table 3). Thus they are suitable objects for eclipse time measurements, and we expect that times of light minimum can be observed with high precision even with the methods of visual and photographic photometry. Therefore, in previous period analyses, the weights chosen for the visual and photographic data and for the photoelectric observations are reasonable. Although this study is mainly based on visual and photographic data, the results should be reliable. 


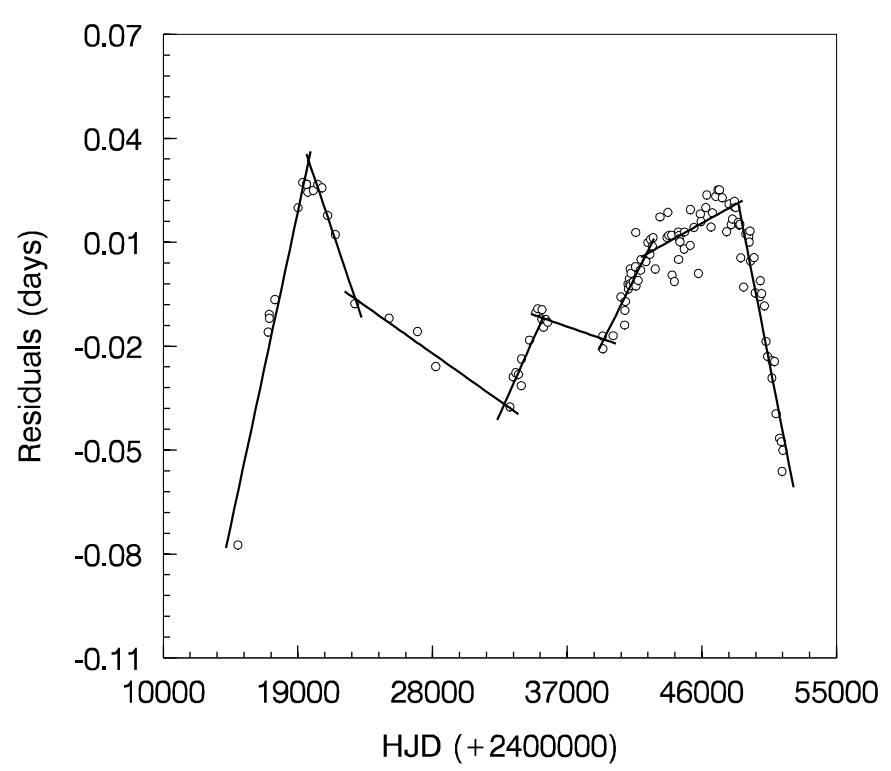

Fig. 5. The same as Fig. 2, but for RR Dra.

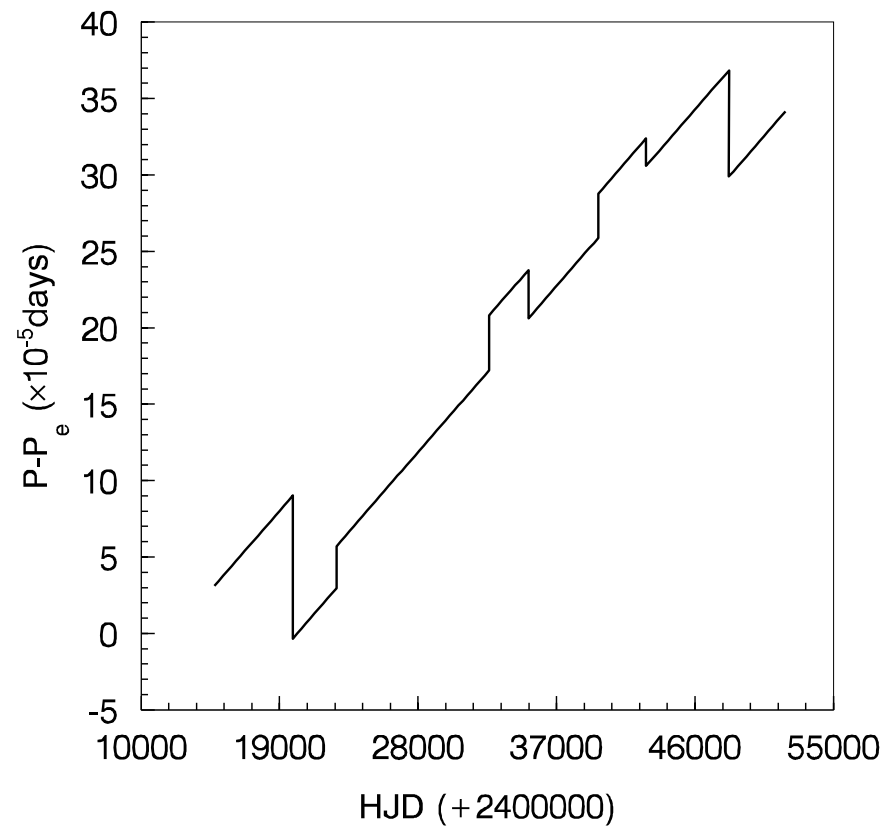

Fig. 6. The same as Fig. 3, but for RR Dra.

The long-term general trends of the $\mathrm{O}-\mathrm{C}$ curves in Figs. 1 and 4 suggest an increasing period for the two binaries, which can be explained as a rapid mass transfer from the less to the more massive components. This is consistent with the semidetached configurations of the systems. The period increase rates are listed in Table 3. By inserting $M_{1}=2.5 M_{\odot}$ and $M_{1}=0.50 M_{\odot}$ given by Mezzetti et al. (1980) to the following equation (Kwee \& van Woerden 1958):

$\Delta P / P=3\left(M_{1} / M_{2}-1\right) \Delta M_{1} / M_{1}$,

the mass transfer rate is determined to be: $\mathrm{d} m / \mathrm{d} t=2.15 \times$ $10^{-7} M_{\odot} /$ year for SW Cyg. For RR Dra, by considering the same parameters as those used for SW Cyg, the mass transfer rate can be estimated to be: $\mathrm{d} m / \mathrm{d} t=3.12 \times 10^{-7} M_{\odot} /$ year.
Table 2. Several irregular period jumps for RR Dra.

\begin{tabular}{lll}
\hline \hline Interval of cycles & $\Delta T$ (days) & $\Delta P\left(10^{-5}\right.$ days $)$ \\
\hline-800 to +1000 & $-0.0210( \pm 0.0031)$ & $+5.73( \pm 0.49)$ \\
+1000 to +2000 & $+0.0681( \pm 0.0059)$ & $-3.63( \pm 0.41)$ \\
+2000 to +5500 & $+0.0114( \pm 0.0083)$ & $-0.87( \pm 0.24)$ \\
+5500 to +6400 & $-0.1890( \pm 0.0203)$ & $+2.73( \pm 0.33)$ \\
+6400 to +8000 & $+0.0156( \pm 0.0061)$ & $-0.43( \pm 0.09)$ \\
+8000 to +9100 & $-0.2134( \pm 0.0199)$ & $+2.47( \pm 0.23)$ \\
+9100 to +11000 & $-0.0542( \pm 0.0144)$ & $+0.68( \pm 0.14)$ \\
+11000 to +12200 & $+0.7172( \pm 0.0502)$ & $-6.27( \pm 0.43)$ \\
\hline
\end{tabular}

Table 3. Secular period change for two Algol-type eclipsing binary systems.

\begin{tabular}{lllllll}
\hline \hline Star name & Sp. & $d$ & $\Delta m$ & Period (days) & $\mathrm{d} P / \mathrm{d} t$ (days/year) & $\tau$ (years) \\
\hline SW Cyg & $\mathrm{A} 2+\mathrm{K} 0$ & 0.11 & 2.6 & 4.5727923 & $4.71 \times 10^{-6}$ & $9.71 \times 10^{5}$ \\
RR Dra & $\mathrm{A} 2+\mathrm{K} 0$ & 0.06 & 3.8 & 2.83110 & $4.24 \times 10^{-6}$ & $6.68 \times 10^{5}$ \\
\hline
\end{tabular}

Since the secular period increases for both binary stars are rather rapid, the mass transfer would be dynamical.

Several photometric and spectroscopic studies have shown that SW Cyg is a low-mass ratio Algol-type binary star $(q=0.20)$ (e.g., Mezzetti et al. 1980). For RR Dra, the primary eclipse is deeper than that of SW Cyg, and both components have the same spectral types as those of SW Cyg. It is expected that its mass ratio may be very low $(q<0.2)$. The rapid period increase suggests that they are low-mass ratio Algol-type binaries in the active phase of mass transfer. According to the evolutionary theory of binary stars, the existence of a slow mass transfer on nuclear timescales is associated with a rather lowmass ratio system. The present study may suggest that a rapid mass exchange still occurs in some of these binaries. These two binaries resemble S Equ (Qian \& Zhu 2002).

By removing the quadratic variations from the $\mathrm{O}-\mathrm{C}$ values, the residuals in Figs. 2 and 4 indicate that several period jumps have occurred. These behaviors are more easily seen in Figs. 3 and 6 where the period changes of the two systems are shown. The period change of SW Cyg displayed in Fig. 3 suggests that, after two consecutive episodes of period increases around HJD 2427100 and HJD 2436700, two successive period decreases took place around HJD 2441800 and HJD 2445900. The same properties in the period change of RR Dra are also found, where two successive episodes of period increases took place around HJD 2422700 and HJD 2432600, and two consecutive period decrease occurred around HJD 2442800 and HJD 2448200. These results suggest that on a short-term timescale the period jumps of the two Algol-type binaries are not alternate, but rather are random. These properties resemble those of U Sge, RW Mon (Šimon 1997a,b), and TW Lac (Qian \& Boonrucksar 2002) where two successive episodes of shortening the orbital period were shown. The explanation of the presence of a third body in both systems can be ruled out because no sine-like variations can be found from the residuals shown in Figs. 2 and 4. 
Hall (1989) found a connection between the spectral type of the binary components and the presence of an alternating period. His study showed that when both components in a binary have a spectral type earlier than F5, the period never displays an alternating change, which was later confirmed by Šimon (1999) and more recently by Zavala et al. (2002). This finding was explained by Hall as evidence of a magnetic activity cycle in the late-type star possessing a convective outer layer (COL). A theory, proposed by Applegate (1992) and by Lanza et al. (1998), explains the alternate period variation by considering the change of internal angular momentum distribution when the active star goes through a magnetic activity cycle. However, the periodic exchange of angular momentum in the COL should cause a strictly alternate change in the period. Are the random period jumps of SW Cyg and RR Dra caused by cyclic magnetic activity?

Since the complicated period changes (alternate or random) only occur in late-type close binaries (i.e., later than F5), it can be concluded that the late-type component should be responsible for such a complex variation (Šimon 1999). An important study by Matese \& Whitmire (1983) indicated that a change of the apsidal motion constant $k_{22}$ should cause a variation in the period. For SW Cyg and RR Dra, instabilities caused by the dynamical mass loss from the K0-type components would cause a change of the distribution of the density $\rho(r)$ in the COL. Thus $k_{22}$ should be variable since it is very sensitive to $\rho(r)$, and therefore the short-term period jumps will occur. The investigation of Gilliland (1985) has shown that the mass loss through the vicinity of the inner Lagrange point $\left(L_{1}\right)$ would result in a decrease of pressure in the neighboring region. This will cause sideways mass motion in COL towards the cone near $L_{1}$, and the remaining part of the K0-type loser will behave like an "infinite" reservoir of mass. The steady period increases in SW Cyg and RR Dra may suggest that a balance in this dynamical process can be reached. Algol-type binaries, showing rapid period increase accompanied by random jumps, may be a very important source helpful to understand interior structura changes of late-type giants or subgiants. More studies on this type of star are required. However, the period changes plotted in Figs. 3 and 6 may show alternate period changes over a very long timescale, especially in Fig. 3 for SW Cyg. The explanation of a magnetic activity cycle in the K0-type component cannot be ruled out.
Acknowledgements. This work was supported by Yunnan Natural Science Foundation (No. 2001A0026Q), the Chinese Natural Science Foundation (No. 10003004), and by the National Key Fundamental Research Project (No. G1999075405).

\section{References}

Agerer, F., Dahm, M., \& Hubscher, J. 2001, IBVS No. 5017

Applegate, J. H., \& Patterson, J. 1987, ApJ, 322, 621

Berrington, R. C., \& Hall, D. S. 1994, AJ, 107, 1868

Biermann, P., \& Hall, D. S. 1973, A\&A, 27, 249

Chaubey, U. S. 1980, Ap\&SS, 67, 129

Dugan, R. S., \& Wright, F. W. 1939, Contribution from the Princeton University Observatory, No. 19

Frieboes-Conde, H., \& Hall, D. S. 1973, A\&AS, 27, 249

Gilliland, R. L. 1985, ApJ, 292, 522

Hall, D. S. 1989, Space Sci. Rev., 50, 219

Hall, D. S., \& Garrison, L. M. 1972, PASP, 84, 552

Hall, D. S., Quarles, T., Roberts, J., \& Whelan, J. A. J. 1979, Acta Astron., 29, 653

Kwee, K. K., \& van Woerden, H. 1958, Bull. Astron. Inst. Netherlands, 12, 357

Lanza, A. F., Rodonò, M., \& Rosner, R. 1998, MNRAS, 296, 893

Matese, J. J., \& Whitmire, D. P. 1983, A\&A, 117, L7

Mezzetti, M., \& Cester, B. 1980, A\&AS, 39, 273

Nijland, A. A. 1917, AN, 203, 403

Olson, E. C. 1981, IBVS, No. 1938

Olson, E. C. 1982, ApJ, 259, 702

Qian, S. B., \& Boonrucksar, S. 2002, New Astron., 7, 435

Qian, S. B., \& Zhu, L. Y. 2002, ApJS, 142, 139

Seares, F. H. 1912, ApJ, 36, 368

Shapley, H. 1913, ApJ, 37, 154

Šimon, V. 1997a, A\&A, 327, 1087

Šimon, V. 1997b, A\&A, 319, 886

Šimon, V. 1999, A\&AS, 134, 1

Struve, O. 1946, ApJ, 104, 253

Szczepanowska, A. 1955, Acta Astron., 5, 74

Szczepanowska, A. 1956, Acta Astron., 6, 144

Szczepanowska, A. 1959, Acta Astron., 9, 46

Walter, K. 1971, A\&A, 13, 249

Whitney, B. S. 1957, AJ, 62, 371

Wilson, R. E., \& Mukherjee, J. 1988, AJ, 96, 747

Yoon, T. S., Honeycutt, R. H., Kaitchuck, R. H., \& Schlegel, E. M. 1994, PASP, 106, 239

Zavala, R. T., McNamara, B. J., Harrison, T. E., et al. 2002, AJ, 123, 450 